\title{
Analysis on Collapse Mechanisms and Weight-efficient of Sandwich Circular Panel
}

\author{
Zhi-Cheng XIA, Jian-Liang ZHANGa , Jing-Yang ZHOU and Nai-Shu ZHU \\ PLA University of Science and Technology, College of Defense Engineering, \\ Nanjing, China \\ azjl18761682179@sina.com
}

Keywords: Sandwich circular panel, Flat-end cylindrical indenter, Collapse mechanism, weightefficient.

\begin{abstract}
Sandwich circular panel with foam core indented by flat-end cylindrical indenter was constructed. Collapse mechanisms and influencing factors of sandwich circular panel was determined, and the mass of sandwich circular panel was lightened through theoretical analysis. The theoretical predictions were verified by comparing with results from commercial code ABAQUS. Results indicate that collapse mechanisms map of sandwich circular panels is similar to beam (panel) in a three-point bend besides the regional boundaries. The maximum material utilization rate of each collapse mechanism is located in the boundaries. The maximum material utilization of all Collapse mechanisms is located in the common point which belong to the face yield mode of collapse, the indentation mode of collapse and the mode B for core shear.
\end{abstract}

\section{Introduction}

Foam-core sandwich panel generally refers to the panel made by two face sheets and one core, which can not only take full use of low density and large deformation of the foam, but also solve the low stiffness of pure foam structure. The collapse mechanisms of foam-core sandwich beam (slab) in a point bend mainly include epidermal yield, core shear, indentation, cohesion failure and face sheet wrinkling. Ashby et al. [1] summarized the collapse mechanisms and analyzed the weight-efficient of aluminum foam sandwich panels indented by square indenter. Shuaeib et al. [2] studied the face yield and the core shear of different polymer sandwich panels indented by circular indenter. Soden [3] assumed the face sheets to be rigid-perfectly plastic and perfectly predicted face yield through linear elastic theory. McCormack et al. [4] drawn the collapse mechanism maps of metallic foam sandwich panels and compared the collapse load with the first peak value of curve. Chen et al. [5] researched the collapse mechanisms and the weight-efficient of metallic foam sandwich panels in a four point bending. Some other analysis on the collapse mechanisms of foam-core sandwich in a three-point bending were carried out by Shang Jintang, Zhang Lin and Wang Erheng and et al in china [6-8].

In summary, much effort on the collapse mechanisms of sandwich rectangular panel (beam) has already been made. Basing on the classic analysis method of the collapse mechanisms, this paper tries to get the collapse mechanisms of foam-core sandwich circular panel indented by flat-end cylindrical indenter through theoretical calculation and numerical simulation. Influencing factors of collapse mechanisms maps and differences of the collapse mechanisms maps between sandwich circular panel and sandwich rectangular beam (panel) are also analyzed. In addition, Collapse load over unit gravity is put forward to lighten the sandwich circular panel.

\section{Analysis on the Collapse Mechanisms}

\section{Analytical Model}

The simply supported sandwich circular panel is loaded by moving the flat-end cylindrical indenter with a diameter $(a<<D)$ as shown in Figure 1. Sandwich circular panel is composed of two face 
sheets and one foam core with thicknesses $t$ and $c$, respectively. The diameter of sandwich circular panel is $D+2 H$ (the diameter amongst the outer supports is $D$ and the overhang distance beyond the outer supports is $H$ ).

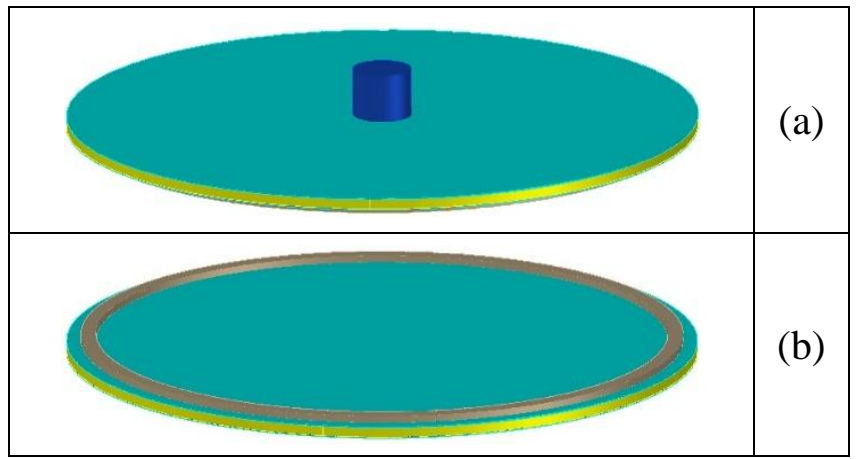

Fig. 1. Three-dimensional diagram of sandwich circular panel (a) the front surface and (b) the rear surface

In order to simplify the discussion and calculation, some assumptions are proposed as follows:

(1) Deformation of the indenter and the outer supports is ignored during the loading process.

(2) The material model of sandwich panels is assumed as rigid-perfectly plastic.

(3) The face sheets and the foam-core are bonded perfectly, and no peeling occurs during the loading process.

\section{Collapse Mechanisms}

\section{Face Yield}

The bending moments over unit length of sandwich panels loaded by transverse concentrated force is written as follows (Contribution of the core is ignored) [9]:

$$
\begin{gathered}
\text { Radial: } M_{R}=\sigma_{R} t(t+c) \\
\text { Tangential: } M_{\theta}=\sigma_{\theta} t(t+c) \\
\text { Yield: } M_{p}=\sigma_{S} t(t+c)
\end{gathered}
$$

Where $\sigma_{R}$ is the radial stress, $\sigma_{\theta}$ is the tangential stress, $\sigma_{S}$ is the constant plateau stress, $t$ is the thickness of the face sheet, and $c$ is the thickness of the core.

Consider the Tresca yield criteria, the maximum among $\left|M_{R}\right|,\left|M_{\theta}\right|$ and $\left|M_{R}-M_{\theta}\right|$ should be equal to $M_{P}$ when the face sheets appear to be yield, as follow:

$$
\operatorname{Max}\left(\left|M_{R}\right|,\left|M_{\theta}\right|,\left|M_{R}-M_{\theta}\right|\right) M_{R}=M_{P}
$$

Note $M_{R} \geq 0, M_{\theta} \geq 0$ and $M_{R} \geq M_{\theta}$ when circular panel is loaded by transverse concentrated force, and we can conclude:

$$
M_{R}=M_{P}, \quad \sigma_{R}=\sigma_{S}
$$

Owing to changes of lateral compress at the radius of $a / 2(a<<D)$, face yield occurs at the radius of $a / 2$. The force equilibrium on the sector of the panel, as sketched in Figure 2(a), gives:

$$
M_{P} \frac{a}{2} \theta=\int_{0}^{\frac{a}{2}} P_{0} \theta r^{2} d r=\frac{\theta F_{1} a^{3}}{6 \pi a^{2}}
$$

Where $P_{0}=4 F_{1} / \pi a^{2}$ is the mean stress applied on the contact region of the upper panel, and the resulting collapse load, $F_{1}$, is given by: 


$$
F_{1}=3 \pi M_{P}=3 \pi \sigma_{S} t(t+c)
$$

\section{Indentation}

The indentation mode of collapse involves the formation of plastic hinges at both the radius of $a / 2$ and the radius of $a / 2+\lambda$ within the top face sheet adjacent to indenter, with compressive yield of the underlying core, as sketched in Figure 2(b). The energy equilibrium gives:

$$
\pi(2 \mathrm{a}+2 \lambda) M_{R} \theta+\left(\frac{\pi}{4} a^{2}+\frac{\pi}{3} \lambda^{2}\right) \theta \lambda \sigma_{Y_{C}}=F_{2} \theta \lambda
$$

Where $M_{R}=\sigma_{S} t^{2} / 4$ is the full plastic moment of the face-sheet section, and the radial wavelength $\lambda$ is rotated through a small angle $\theta$. The resulting collapse load, $F_{2}$, is given by:

$$
F_{2}=2 \pi\left(\frac{\mathrm{a}}{\lambda}+1\right) \frac{\sigma_{S} t^{2}}{4}+\pi\left(\frac{1}{4} a^{2}+\frac{1}{3} \lambda^{2}\right) \sigma_{Y_{C}}
$$

The principle of virtual work tells us that the actual value $\lambda$ among many possible values that meet the Eq. (9) should make collapse load, $F_{2}$, minimal. That is, the variation of $F_{2}$ is divided into zero.

$$
\begin{gathered}
\delta F_{2}=0 \\
\lambda=\sqrt[3]{\frac{3 a \sigma_{S} t^{2}}{4 \sigma_{Y c}}} \\
F_{2}=\frac{3 \pi \sigma_{S} t}{4} \sqrt[3]{\frac{4 a^{2} t \sigma_{Y c}}{3 \sigma_{S}}}+\frac{\pi \sigma_{S} t^{2}}{2}+\frac{\pi}{4} a^{2} \sigma_{Y c}
\end{gathered}
$$

\section{Core Shear}

The core shear mode of collapse is divided into mode A and mode B due to the overhang length $H$, as sketched in Figure 2(c) and Figure 2(d). Mode A comprises plastic hinge formation at the radius of $a / 2$, with shear yielding of the core. The energy equilibrium gives:

$$
2 \pi \mathrm{a} M_{R} \theta+\pi\left(\frac{D}{2}+H\right)^{2} \theta c \tau_{Y C}=F_{3 A} \frac{D \theta}{2}
$$

Where $M_{R}=\sigma_{S} t^{2} / 4$ the full plastic moment of the face-sheet is section, and $\tau_{Y C}$ is the shear yield strength for the core. The resulting collapse load, $F_{3 A}$, is given by:

$$
F_{3 A}=\frac{\pi a \sigma_{S} t^{2}}{D}+\frac{\pi}{2 D}(D+2 H)^{2} c \tau_{Y C}
$$

Mode $\mathrm{B}$ comprises plastic hinge formation at both the radius of $a / 2$ and the radius of $D / 2$, with shear yielding of the core amongst the outer supports. The energy equilibrium gives:

$$
2 \pi(\mathrm{a}+D) M_{R} \theta+\pi \frac{D^{2}}{4} \theta c \tau_{Y c}=F_{3 B} \frac{D \theta}{2}
$$

Where $M_{R}=\sigma_{S} t^{2} / 4$ is the full plastic moment of the face-sheet section, and the result collapse load is given by

$$
F_{3 B}=\pi\left(\frac{\mathrm{a}}{D}+1\right) \sigma_{S} t^{2}+\frac{\pi D}{2} c \tau_{Y C}
$$

The transition length of overhang, $H_{t}$, from Mode A to Mode B is determined by equating Equal Eqs. (14) and (16), giving: 


$$
H_{t}=\frac{D}{2}\left(\sqrt{1+\frac{2 \sigma_{S} t^{2}}{D c \tau_{Y C}}}-1\right)
$$

\section{Collapse Mechanism Maps}

To simplify the analysis, a formula of non-dimensional collapse load is given by Eq. (18). The operative collapse mechanism of the sandwich circular panel is the one associated with the lowest non-dimensional collapse load [1]. The collapse mechanisms and the corresponding non-dimensional collapse loads of sandwich circular panel are listed in Table 1.

$$
\bar{F}=4 F / \pi \sigma_{S} D^{2}
$$

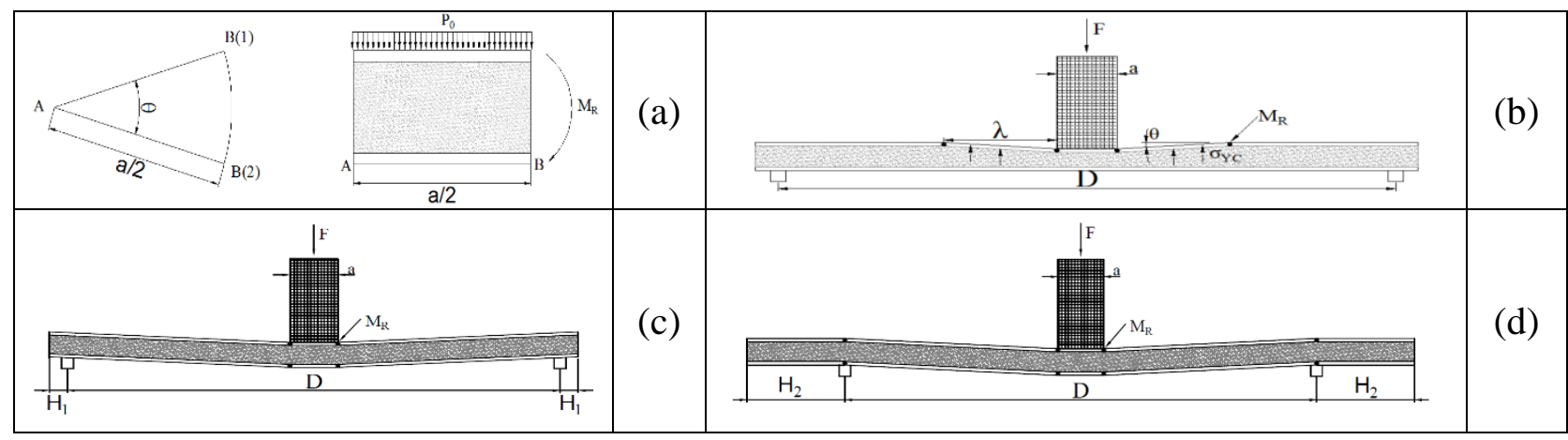

Fig. 2. The sandwich circular panels (a) the balance sector of face yield, (b) indentation mode of collapse, (c) mode A for core shear and (d) mode B for core shear

Table 1. Non-dimensional collapse load and the simplified CLUG

\begin{tabular}{|c|c|c|}
\hline $\begin{array}{c}\text { Collapse } \\
\text { mechanisms }\end{array}$ & $\begin{array}{c}\text { The non-dimensional } \\
\text { collapse load (1) }\end{array}$ & The simplified bearing force over unit gravity (1) \\
\hline Face yield & $\overline{F_{1}}=12\left(\frac{t}{D} \frac{t}{D}+\frac{c}{D} \frac{t}{D}\right)$ & $\bar{f}_{1}=12 \frac{t}{D}\left(\frac{t}{D}+\frac{c}{D}\right) /\left(\frac{\rho_{C}}{\rho_{S}} \frac{c}{D}+\frac{t}{D}\right)\left(1+2 \frac{H}{D}\right)^{2}$ \\
\hline Indentation & $\overline{F_{2}}=3 \frac{\mathrm{t}}{D} \sqrt[3]{\frac{4 a^{2} \sigma_{Y C}}{3 D^{2} \sigma_{S}} \frac{\mathrm{t}}{D}}+2 \frac{t^{2}}{D^{2}}+\frac{a^{2}}{D^{2}} \frac{\sigma_{Y c}}{\sigma_{S}}$ & $\bar{f}_{2}=\left(3 \frac{t}{D} \sqrt[3]{\frac{4 a^{2} \sigma_{Y C}}{3 D^{2} \sigma_{S}} \frac{t}{D}}+2 \frac{t^{2}}{D^{2}}+\frac{\sigma_{Y C}}{\sigma_{S}} \frac{a^{2}}{D^{2}}\right) /\left(\frac{\rho_{C}}{\rho_{S}} \frac{c}{D}+\frac{t}{D}\right)\left(1+2 \frac{H}{D}\right)^{2}$ \\
\hline $\begin{array}{c}\text { Mode A for } \\
\text { core shear }\end{array}$ & $\overline{F_{3 A}}=4 \frac{a}{D} \frac{t^{2}}{D^{2}}+2\left(1+2 \frac{H}{D}\right)^{2} \frac{c}{D} \frac{\tau_{Y c}}{\sigma_{S}}$ & $\bar{f}_{3 A}=\left(\frac{4 a}{D} \frac{t^{2}}{D^{2}}+2 \frac{\tau_{Y C}}{\sigma_{S}}\left(1+2 \frac{H}{D}\right)^{2} \frac{c}{D}\right) /\left(\frac{\rho_{C}}{\rho_{S}} \frac{c}{D}+\frac{t}{D}\right)\left(1+2 \frac{H}{D}\right)^{2}$ \\
\hline $\begin{array}{c}\text { Mode B for } \\
\text { core shear }\end{array}$ & $\overline{F_{3 B}}=4\left(\frac{\mathrm{a}}{D}+1\right) \frac{t}{D} \frac{t}{D}+2 \frac{c}{D} \frac{\tau_{Y C}}{\sigma_{S}}$ & $\bar{f}_{3 B}=\left(4\left(\frac{a}{D}+1\right) \frac{t^{2}}{D^{2}}+\frac{2 \tau_{Y c}}{\sigma_{S}} \frac{c}{D}\right) /\left(\frac{\rho_{C}}{\rho_{S}} \frac{c}{D}+\frac{t}{D}\right)\left(1+2 \frac{H}{D}\right)^{2}$ \\
\hline
\end{tabular}

\section{Collapse Mechanism Maps of the Core}

To gage the practical significance of the overhang, let us take some representative values for the sandwich circular panel comprising steel skins and an aluminum foam core, with $\tau_{Y c} / \sigma_{Y c}=0.667$, $\sigma_{Y c} / \sigma_{S}=0.005, t / D=0.01, c / \mathrm{D}=0.1, a / D=0.1$. Then the transition overhang length, $H_{t}$, is given by $H_{t}=0.132 D$, and relationship between $\bar{F}_{3}$ and $H / D$ is given in Figure 3(a). Figure 3(b) is the relationship between $\bar{F}_{3}$ and $H / D$ of sandwich rectangular panel from the reference [1]. When the overhang length $H$ is smaller than the transition overhang length $H_{t}$, the non-dimensional collapse load $\overline{F_{3 A}}$ of mode $\mathrm{A}$ has a quadratic relationship with $H / D$ in Figure 3(a), and a linear relationship with $H / D$ in Figure 3(b). When the overhang length $H$ is larger than the transition overhang length $H_{t}$, the non-dimensional collapse load $\overline{F_{3 B}}$ of mode B has no relationship with $H / D$ in Figure 3(a) and Figure 3(b). 


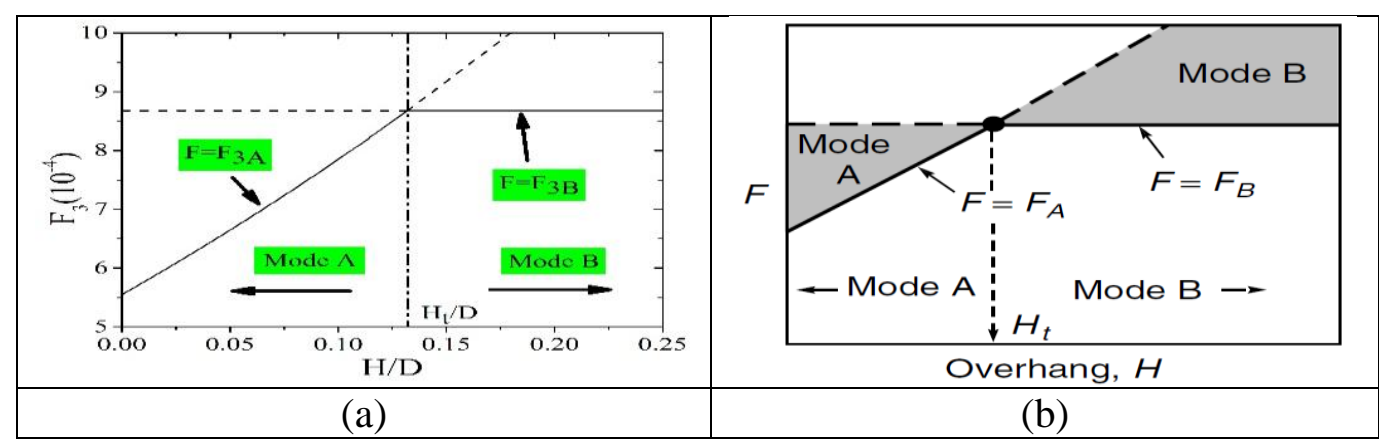

Fig. 3. The relationship between collapse load for core shear and $H / D$ of (a) sandwich circular panel and (b) sandwich rectangular panel (beam)

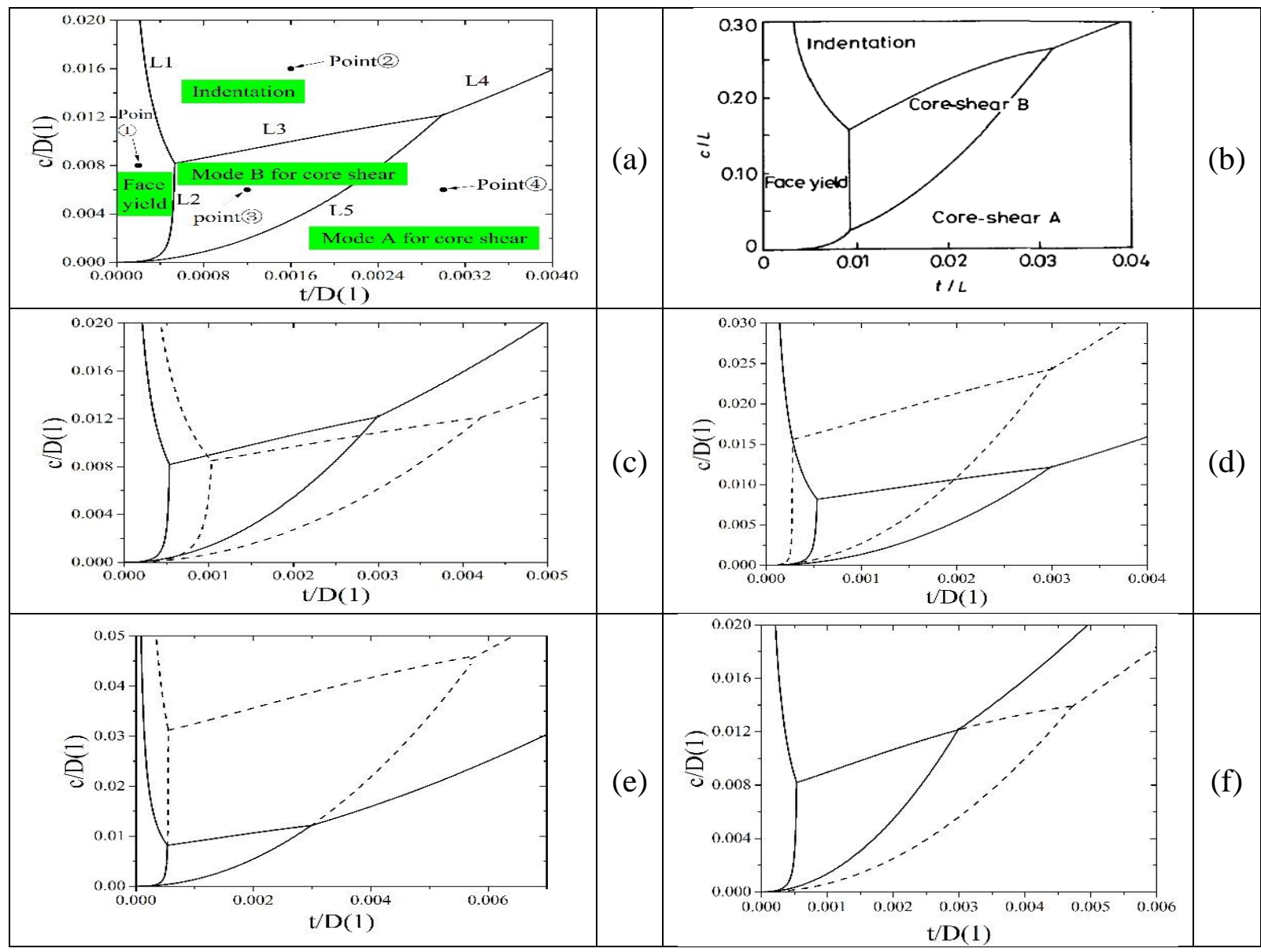

Fig. 4. Collapse mechanisms maps of (a) Sandwich circular panel and (b) sandwich rectangular panel (beam) (c) $\sigma_{Y c} / \sigma_{S}=0.010$, (d) $\tau_{Y c} / \sigma_{Y c}=0.333$,(e) $a / D=0.2$ and (f) $H / D=0.2$

\section{Collapse Mechanism Maps of the Sandwich Circular Panel and Influencing Factors}

An example of collapse mechanism map for sandwich circular panel is given in Figure 4(a) with $H / D=0.1, \sigma_{Y c} / \sigma_{S}=0.005, \tau_{Y c} / \sigma_{Y C}=0.667$ and $a / D=0.1$. Figure $4(\mathrm{~b})$ is the collapse mechanism map of sandwich rectangular panel (beam) from reference [7]. Many common characteristics besides the boundaries can be found by comparing Figure 4(a) with Figure 4(b). The influence of variables including $\tau_{Y c} / \sigma_{Y c}, \sigma_{Y C} / \sigma_{S}, a / D$, and $H / D$ on the collapse mechanism maps of sandwich circular panel is further studied by the method of control variable, as shown in Table 2.

The solid lines plotted in Figure 4(c), Figure 4(d), Figure 4(e), and Figure 6(f) are identical with those in Figure 4(a). The dotted lines are drawn with changed values of influencing factors given in 
Table 2. The area of mode $\mathrm{A}$ increases and the areas of the others decrease when the value of $\sigma_{Y C} / \sigma_{S}$ increases, as shown in Figure 4(c). The areas of mode A and mode B increase and the areas of the others decrease when the value of $\tau_{Y c} / \sigma_{Y c}$ decreases, as shown in Figure 4(d). The area of the indentation decreases and the areas of the others increase when the value of $a$ / D increases, as shown in Figure 4(e). The area of mode A increases, the area of the face yield is invariant and the areas of the others decrease when the value of $H / D$ increases, as shown in Figure 4(f).

Table 2. The influencing factors and values

\begin{tabular}{|c|c|c|c|c|}
\hline plans & $\sigma_{Y c} / \sigma_{S}$ & $\tau_{Y c} / \sigma_{Y c}$ & $a / D$ & $H / D$ \\
\hline (c) & 0.010 & 0.667 & 0.1 & 0.1 \\
\hline (d) & 0.005 & 0.333 & 0.1 & 0.1 \\
\hline (e) & 0.005 & 0.667 & 0.2 & 0.1 \\
\hline (f) & 0.005 & 0.667 & 0.1 & 0.2 \\
\hline
\end{tabular}

\section{Analysis on the Weight-Efficient}

\section{Collapse Load over Unit Gravity (CLUG for Short)}

In order to make full use of materials and meet the requirements of lightweight sandwich circular panel, the CLUG is proposed as a weight index, as follow:

$$
\begin{gathered}
f=\frac{F}{M g} \\
\bar{f}=\frac{g D \rho_{S}}{\sigma_{S}} f
\end{gathered}
$$

Where $f$ is the CLUG, $\bar{f}$ is the simplified formula of $f, M=\pi(D+2 H)^{2}\left(\rho_{C} c+\rho_{S} t\right) / 4$ is the total body mass of sandwich circular panel, $F$ is collapse load, and $g$ is the acceleration of gravity.

\section{Optimization on the CLUG}

Substituting Eqs. (7), (12), (14) and (16) into Eq. (20), the simplified CLUG of each collapse mechanisms can be obtained, as listed in Table1. According to the example given in section 2.3 .2 and $\rho_{C} / \rho_{S}=0.043$, the change trend of simplified CLUG in each collapse mechanism region is analyzed as follows:

$$
\left\{\begin{array}{l}
d\left(\bar{f}_{1}\right) / d(t / D) \geq 0 \\
d\left(\bar{f}_{1}\right) / d(c / D) \geq 0
\end{array}\right.
$$

From Eq. (21), we can obtain that the maximum simplified CLUG of the face yield region is located in the right boundaries or the upper boundaries.

$$
\left\{\begin{array}{c}
\text { make } d\left(\bar{f}_{2}\right) / d(t / D)=0 \\
d\left(\bar{f}_{2}\right) / d(c / D)<0
\end{array}\right.
$$

From Eq. (22), we can obtain that the minimum simplified CLUG of the indentation region is located in the line of $/ D=\left(2.887 \times 10^{-4}-11.547(t / D)^{2}\right) /(t / D-0.041 \sqrt[3]{t / D})$. When the value of $t / D$ increases, the simplified CLUG decreases in the left region of the line, and that is contrary in the right region of the line. The maximum simplified CLUG of the indentation region is located in the left lower boundaries or the right lower boundaries. 


$$
\left\{\begin{array}{l}
\text { make } d\left(\bar{f}_{3 A}\right) / d(t / D)=0 \\
\text { make } d\left(\bar{f}_{3 A}\right) / d(c / D)=0
\end{array}\right.
$$

From Eq. (23), we can obtain that the minimum simplified CLUG of the mode A region is located in the point of $\left(5.546 \times 10^{-2}, 1.602 \times 10^{-1}\right)$. When the value of $c / D$ increases, the simplified CLUG increases in the left region of the line described by the formula of $\mathrm{t} / D=5.546 \times 10^{-2}$, and that is contrary in the right region of the line. The maximum simplified CLUG of the mode A region is located in the left upper boundaries or the right lower boundaries.

$$
\left\{\begin{array}{l}
\text { make } d\left(\bar{f}_{3 B}\right) / d(t / D)=0 \\
\text { make } d\left(\bar{f}_{3 B}\right) / d(c / D)=0
\end{array}\right.
$$

From Eq. (24), we can obtain that the minimum simplified CLUG of the mode B region is located in the point of $\left(3.499 \times 10^{-2}, 3.079 \times 10^{-1}\right)$. When the value of $c / D$ increases, the simplified CLUG increases in the left region of the line described by the formula of $t / D=3.499 \times 10^{-2}$, and that is contrary in the right region of the line. The maximum simplified CLUG of the mode B region is located in the left upper boundaries.

In summary, the maximum simplified CLUG of each collapse mechanisms region is located in the boundaries. In view of practical significance of this example, four boundaries, that is $c / D=0$, $c / D=+\infty, t / D=0$ and $t / D=+\infty$, are removed. The remaining five boundaries of line 1 , line 2 , line 3, line 4 and line 5 are shown in Figure 6(a). Substituting the formula of line 1, line 2, line 3, line 4 and line 5 into the Eq. (20), respectively, and the results are listed in Table 3.

Table 3. The simplified CLUG of line 1, line 2, line 3, line 4 and line 5

\begin{tabular}{|c|c|}
\hline Boundaries & The simplified CLUG $/ 1$ \\
\hline Line 1 & $\bar{f}_{L 1}=\frac{1.470 \frac{t^{3}}{D^{3}}+3.740 \times 10^{-5} \frac{t}{D}+0.216 \frac{t^{2}}{D^{2}} \sqrt[3]{\frac{t}{15 D}}}{\frac{t^{2}}{D^{2}}+1.872 \times 10^{-7}+1.124 \times 10^{-3} \frac{t}{D} \sqrt[3]{\frac{t}{15 D}}}$ \\
\hline Line 2 & $\bar{f}_{L 2}=\frac{8.333 \frac{t}{D}-5500 \frac{t^{2}}{D^{2}}}{1-1750.638 \frac{t}{D}}$ \\
\hline Line 3 & $\bar{f}_{L 3}=\frac{1.389 \frac{t^{2}}{D^{2}}+3.472 \times 10^{-5}+0.208 \frac{t}{D} \sqrt[3]{\frac{t}{15 D}}}{\frac{t}{D}-15.588 \frac{t^{2}}{D^{2}}+3.248 \times 10^{-4}+1.949 \frac{t}{D} \sqrt[3]{\frac{t}{15 D}}}$ \\
\hline Line 4 & $\bar{f}_{L 4}=\frac{1.389 \frac{t^{2}}{D^{2}}+3.474 \times 10^{-5}+0.208 \frac{t}{D} \sqrt[3]{\frac{t}{15 D}}}{\frac{t}{D}-7.217 \frac{t^{2}}{D^{2}}+2.256 \times 10^{-4}+1.354 \frac{t}{D} \sqrt[3]{\frac{t}{15 D}}}$ \\
\hline Line 5 & $\bar{f}_{L 5}=\frac{9.368 \frac{t}{D}}{1+59.045 \frac{t}{D}}$ \\
\hline
\end{tabular}




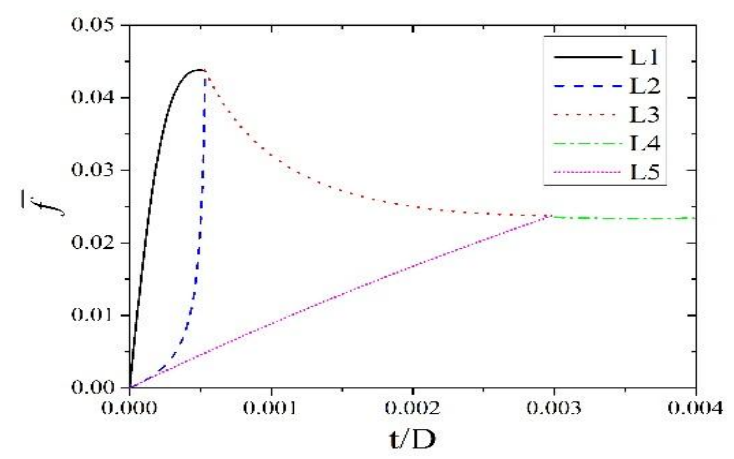

Fig. 5. Relationship between the CLUG and $t / D$

Figure 5 is drawn according to the Table 3 and Figure 6(a). From the Figure 5, we can find the maximum simplified CLUG of all collapse mechanisms is located in the intersection point of line 1, line 2 and line 3 , in which the utilization rate of the material is also maximum.

These parameters is selected by random, so this conclusion can be popularized to the common situations.

\section{Numerical Simulation}

To verify the validity and applicability of the present analytical model and the indentation force predicted by proposed model, the results of which is compared with those calculated by the commercial code ABAQUS.

\section{Finite Element Model}

The finite element model involves indenter and sandwich circular panel, as shown in Figure 6. The dimensions of the model are consistent with the actual components. Owing to the sandwich circular panel indented by a flat-end cylindrical indenter, an axisymmetric model can be constructed. The indenter is modeled as a rigid body and the core and the face sheets are simulated by a 4-node bilinear axisymmetric quadrilateral element with reduced integration (CAX4R) [10]. The matrix materials of the core and face sheet are aluminum foam and steel, respectively, as listed in Table4. Unit system using kg-m-s. To prevent delamination between face sheet and core, and force transferred from face sheet to core through co-node method. The contact between the indenter and upper face sheet is simulated by SURFACE TO SURFACE CONTACT (Standard), tangential friction of all contact surfaces is simulated by the penalty formula, and friction coefficient is 0.3 . Meshing size of the model is $1 \mathrm{~mm}$.

The sandwich circular panel is simply supported on the periphery. Pressure loading is realized by changing displacement boundary condition of the indenter.

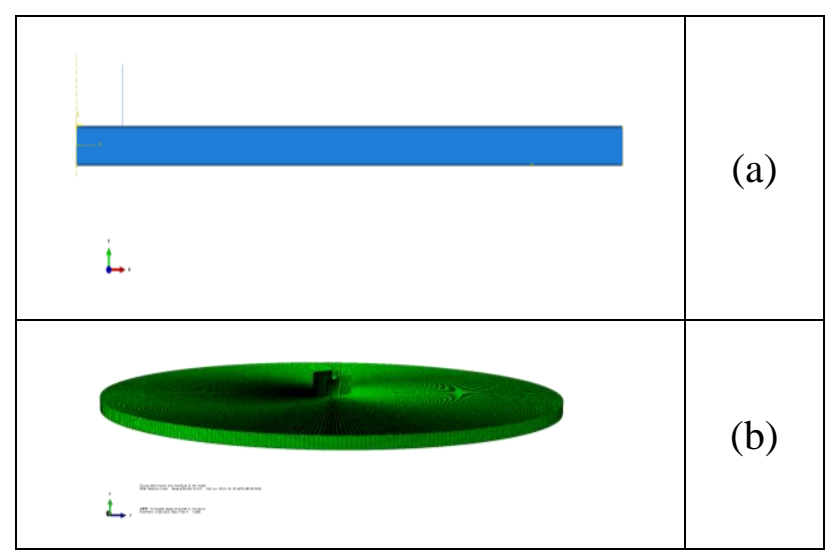

Fig. 6. The finite element model of (a) axisymmetric model and (b) overall model 
Table 4. Parameters and values of matrix material

\begin{tabular}{|c|c|c|c|c|}
\hline Material & $\rho_{0} /(\mathrm{kg} \cdot \mathrm{m}-3)$ & $E / \mathrm{MPa}$ & $v$ & $\sigma_{Y} / \mathrm{MPa}$ \\
\hline Steel & $7.85 \mathrm{E} 3$ & $2.01 \mathrm{E} 5$ & 0.3 & $2.85 \mathrm{E} 2$ \\
\hline Foam & $3.40 \mathrm{E} 2$ & $3.80 \mathrm{E} 2$ & 0.0 & $1.43 \mathrm{E} 0$ \\
\hline
\end{tabular}

\section{Simulation Schemes and Results}

According to the examples given in section 2.3.2, we choose $\sigma_{S}=285 \mathrm{MPa}$ and $D=2 \mathrm{~m}$, then $\tau_{Y c}=$ $0.95 \mathrm{MPa}, \quad a=0.2 \mathrm{~m}, H=0.2 \mathrm{~m}$. Four points which belong to different collapse mechanisms are selected from Figure 4(a), and the simulations are carried out. Results of the simulation are presented in Table 5, and the four corresponding deflection-collapse load curves are plotted in Figure 7. The relationship between the peak value, $f_{R P}$, of the curve in Figure 7 and the collapse load, $F$, in Table 5 is given by

Table 5. Schemes and results of numerical simulation

\begin{tabular}{|c|c|c|c|c|c|c|c|c|c|c|}
\hline \multirow{2}{*}{$\stackrel{0}{\stackrel{0}{E}}$} & \multicolumn{2}{|c|}{ Dimension } & \multicolumn{5}{|c|}{ Collapse load } & \multicolumn{2}{|c|}{$\begin{array}{c}\text { Collapse } \\
\text { mechanisms }\end{array}$} & \multirow{2}{*}{$\frac{\pi}{0}$} \\
\hline & $t / D$ & $c / D$ & $F_{1} / \mathrm{KN}$ & $F_{2} / \mathrm{KN}$ & $\begin{array}{l}F_{3 A} \\
/ \mathrm{KN}\end{array}$ & $\begin{array}{l}F_{3 B} \\
/ \mathrm{KN}\end{array}$ & $F / \mathrm{KN}$ & $\begin{array}{l}\text { Predict- } \\
\text { ed }\end{array}$ & Virtual & \\
\hline (1) & 0.0002 & 0.008 & 17.6116 & 46.0898 & 68.7426 & 47.8855 & 16.8563 & $\begin{array}{l}\text { Face } \\
\text { yield }\end{array}$ & $\begin{array}{l}\text { Face } \\
\text { yield }\end{array}$ & 4.29 \\
\hline$(2)$ & 0.0016 & 0.016 & $\begin{array}{c}302.404 \\
6\end{array}$ & 69.6985 & $\begin{array}{c}138.373 \\
0\end{array}$ & $\begin{array}{c}105.536 \\
2\end{array}$ & 65.3600 & $\begin{array}{l}\text { Indenta } \\
\text { tion }\end{array}$ & $\begin{array}{c}\text { Indentati } \\
\text { on }\end{array}$ & 6.22 \\
\hline (3) & 0.0012 & 0.006 & 92.7832 & 61.2039 & 52.0617 & 41.4661 & $\begin{array}{c}41.7868 \\
1\end{array}$ & $\begin{array}{c}\text { Mode B } \\
\text { for core } \\
\text { shear }\end{array}$ & $\begin{array}{c}\text { Mode B } \\
\text { for core } \\
\text { shear }\end{array}$ & 0.77 \\
\hline (4) & 0.0030 & 0.006 & $\begin{array}{c}289.947 \\
6\end{array}$ & $\begin{array}{c}107.953 \\
9\end{array}$ & 54.7679 & 71.2340 & 50.6928 & $\begin{array}{l}\text { Mode } \\
\text { A for } \\
\text { core } \\
\text { shear }\end{array}$ & $\begin{array}{c}\text { Mode A } \\
\text { for core } \\
\text { shear }\end{array}$ & 7.44 \\
\hline
\end{tabular}

Where point(1), point(2), point(3) and point(4) is signed in Fig.6(a)

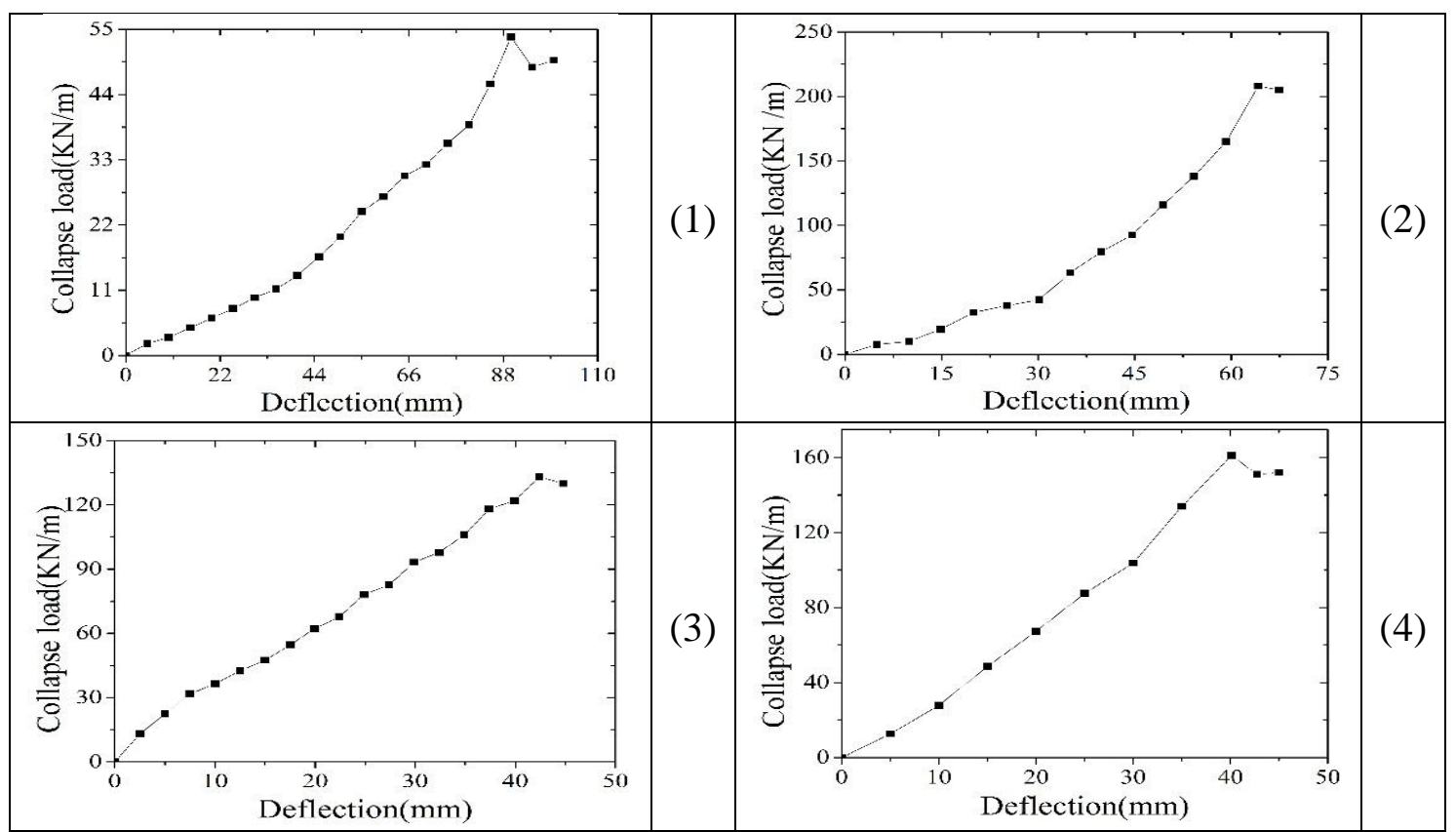

Fig. 7. Deflection-collapse load curve of (1)face yield mode of collapse, (2)indentation mode of collapse, (3) mode B for core shear and (4) mode A for core shear 


$$
F=0.314 \times f_{R P}
$$

From the Table 5, it is known that the results of simulation are in agreement with the theoretical predictions, and the error is less than $7.5 \%$.

\section{Conclusions}

In this paper, the collapse mechanisms and weight-efficient of sandwich circular panel indented by the flat-end cylindrical indenter are analyzed. Specific conclusions are given as follows:

(1) Many common characteristics besides the boundaries can be found between sandwich circular panel and sandwich rectangular panel (beam).

(2)The area of mode $\mathrm{A}$ increases and the area of the others decrease when the value of $\sigma_{Y c} / \sigma_{S}$ increases. The areas of mode A and mode B increase and the areas of the face yield and the indentation decrease when the value of $\tau_{Y C} / \sigma_{Y C}$ decreases. The area of the indentation decreases and the areas of the others increase when the value of $a$ /D increases. The area of mode A increases, the area of the face yield is invariant and the areas of the others decrease when the value of $H / D$ increases.

(3) The simplified CLUG is proposed to lighten the weight of the sandwich circular panel. The maximum simplified CLUG of each collapse mechanism is located in the boundaries. In view of practical significance of problem, the maximum simplified CLUG of all collapse mechanism is located in the intersection point which belong to the face yield mode of collapse, the indentation mode of collapse and the mode B for core shear.

\section{Acknowledgments}

This work is supported by the National Natural Science Foundation of China (51478469) and outstanding young investigator award (51505497)

\section{References}

1. Ashby M F, Evans R M, Fleck N A, et al. Metal Form: A Design Guide [M]. Boston: ButterworthHeinemann, 2000.

2. Shuaeib F M., Soden P D, Indentation failure of composite sandwich beams [J]. Composite Science and Technology, 1997, 57(9-10):1249- 1259.

3. Soden P D. Indentation of composite sandwich beams [J]. Journal of Strain Analysis for Engineering Design, 1996, 31(5):353-360.

4. McCormack T M, 'Miller R, Kesler O, et al. Failure of sandwich beams with metallic foam cores[J]. International Journal of Solids and Structures 2001: 38(28-29): 4901-4920.

5. Chen C, Harte A M, Fleck N A. The plastic collapse of sandwich beams with a metallic foam core [J]. International Journal of Mechanical Sciences, 2001, 43(6): 1483- 1506.

6. SHANG Jin-tang, HE De-ping. Deformation of sandwich beams with Al foam cores in threepoint beading [J]. Chinese Journal of Materials research, 2003, 17(1): 31-38.

7. ZHANG Lin, HE De-ping. Deformation of sandwich beams with spherical pore Al alloy foam core in three-point bending [J]. Chinese Journal of Materials research, 2005, 19(4): 361-368.

8. WANG Er-heng. Study on impact behaviour of foamed aluminium and aluminium foam sandwich beams [D]. HE Fei: Doctoral Dissertation of University of Science \&Technology China, 2005.

9. XU Bing-ye, LIU Xin-sheng. Application of elastic and plastic mechanics [M]. Tsinghua University Press, 1993. 
10. MU Lin, CHO Chong-du, ZHAO Gui-ping, et al. A new indentation model for sandwich circular panels with gradient metallic foam cores [J]. Composites Part B, 2015, 83(9):270-275. 